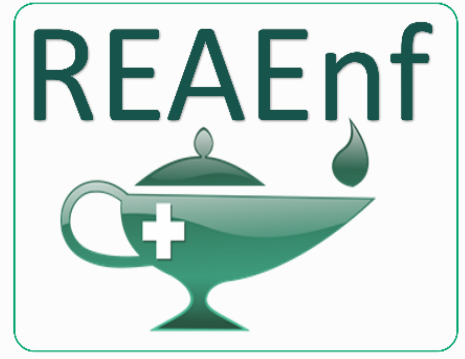

Revista Eletrônica Acervo Enfermagem
ARTIGO ORIGINAL

Recebido em: $3 / 2020$

Aceito em: $4 / 2020$

Publicado em: 6/2020

\title{
Avaliação das gestantes com relação as orientações e cuidados recebidos sobre o parto seguro
}

\author{
Pregnant evaluation regarding the safe childbirth guidance and care received
}
Evaluación de mujeres embarazadas con respecto a la orientación y la atención recibida en el parto seguro

Eloise Natane da Silva ${ }^{1}$, Cristiane Magri da Silva ${ }^{1}$, Daisy Machado ${ }^{1}$, Silmara Alves de Souza ${ }^{1 *}$.

\begin{abstract}
Resumo: Esse artigo avaliou o conhecimento e informações passadas pelos profissionais da saúde com relação ao parto seguro. Portanto realizou-se um estudo quantitativo num Hospital Universitário de Bragança Paulista, através da análise de um questionário, que teve a participação de 108 gestantes, sobre parto seguro. Os resultados demostraram que as gestantes estão com o número de consultas pré-natal em dia, porém $28 \%$ delas dizem saber o que é parto seguro e considerando somente estas $57,1 \%$ relataram conhecer porque tiveram esta informação com profissional da saúde e os outros $42,9 \%$ relatam terem procurado tal informação por conta própria. Contudo quase todas $(92,6 \%)$ relataram que os profissionais escutam suas queixas e tiram dúvida. Conclui-se a partir dos resultados encontrado que existe a necessidade de reformular as informações dadas as gestantes durante as consultas pré-natal principalmente abordar assuntos ainda não colocados em pauta, mas que já se fazem fundamentais na vivência do pré-natal, como no caso o parto seguro.
\end{abstract}

Palavras-chave: Saúde materno-infantil, Parto humanizado, Gravidez.

Abstract: This article evaluate knowing and information provided by health's professionals regarding safe childbirth. Therefore, a quantitative study was developmented in Bragança Paulista's University Hospital, through the questionnaire analysis, which was attended by 108 pregnant, about safe childbirth. The results showed that pregnant have the prenatal number up to data, but $28 \%$ of them said know about safe childbirth and considering only them $57.1 \%$ reported knowing because health professionals explaining and others $42.9 \%$ report having sought such information on their own. However, almost all (92.6\%) reported that professionals listen their complaints and ask questions. It is concluded that there is a need to reformulate the information given to pregnant during prenatal consultations, especially addressing issues not yet approached, but which are already fundamental in the experience of prenatal care, such as safe childbirth.

Keywords: Maternal and child health, Humanizing delivery, Pregnancy.

${ }^{1}$ Universidade São Francisco (USF), Bragança Paulista - SP. *E-mail: silmaraas@bol.com.br 
Resumen: Este artículo buscaba evaluar el conocimiento y la información proporcionada por los profesionales de la salud con respecto a la entrega segura. Por lo tanto, se realizó estudio cuantitativo en un Hospital Universitario en Bragança Paulista, a través del análisis de un cuestionario, al que asistieron 108 mujeres embarazadas, sobre el parto seguro. Los resultados mostraron que las mujeres embarazadas tienen el número de consultas prenatales actualizadas, pero el $28 \%$ de ellas dicen que saben lo que es un parto seguro y consideran que solo estas son las mismas, el $57.1 \%$ informaron saber porque tenían información con profesionales de la salud y otras personas $42.9 \%$ informa haber buscado dicha información por su cuenta. Sin embargo, casi todos (92.6\%) informaron que los profesionales escuchan sus quejas y hacen preguntas. Se concluye que existe la necesidad de reformular la información brindada a las mujeres embarazadas durante las consultas prenatales, especialmente abordando temas que aún no están en la agenda, pero que ya son fundamentales en la experiencia prenatal, como el parto seguro.

Palabras clave: Salud materno-infantil, Parto humanizado, Embarazo.

\section{INTRODUÇÃO}

Um parto seguro e humanizado é caracterizado por uma gestação tranquila e saudável, onde a parturiente e o neonato estarão numa condição mais saudável possível, com o mínimo de intervenção para um momento mais seguro (VELHO MB, 2010; OMS, 2015).

O parto é um evento previsível e complexo, em que várias etapas são necessárias para se chegar bem neste momento, porém muitas vezes é difícil e complicado, portanto, é importante assegurar que tanto a mãe quanto o recém-nascido receberão os cuidados mais seguros possíveis. A maioria das pessoas, que não são profissionais da saúde, considera difícil lembrar-se simplesmente de toda a informações relevantes, como se a bolsa rompeu, horário do início das contrações, em que momento houve a ingesta de medicamento, caso tenha ocorrido, portanto cumprir todos os passos corretamente e na ordem certa é um desafio ainda maior para o desenvolvimento de um parto seguro (GAWANDE A, 2010).

Mesmo tomando todos os cuidados e orientações dadas pelos profissionais da saúde, durante a gestação e o parto, ainda existem inseguranças que são decorrentes a estes momentos como que tipo de parto ocorrerá (normal, cesárea, humanizado), a existência de hemorragias e infecções que podem causar complicações tanto para a gestante como para o feto (OMS, 2017).

Portanto, apesar de o parto ser um conjunto de eventos previsíveis pode ocorrer complicações que podem deixar a vida da mãe e do recém-nascido, ou de ambos, em risco. Dados reportam que por ano são mais de 130 milhões de nascimento, sendo que cerca de $0,25 \%$ desses resultam na morte da parturiente, $2 \%$ natimortos e $2,1 \%$ o recém-nascido morre nos primeiros 28 dias de vida, sendo que a maioria destas mortes poderiam ser evitadas, uma vez que ocorrem na sua maioria devido a baixos recursos (OMS, 2017). Por outro lado, em pleno século 21, com tanta informação e tecnologia, as cifras ainda surpreendem e são um alerta para quem atua na área (RISCADO LC, et al., 2016).

O atendimento de má qualidade durante os nascimentos nos países de baixa e média renda é um dos principais fatores que contribuem para o problema materno e do recém-nascido, mas o progresso tem sido lento na identificação de métodos eficazes para enfrentar essas deficiências em escala. Devido a esta característica a Organização Mundial da Saúde (OMS) liderou o projeto de campo da Lista de Verificação de Parto Seguro do WHO - uma ferramenta de 29 itens que visa levantar as principais causas de mortalidade materna e neonatal globalmente para suprir tais fatores. Sendo assim, a equipe multiprofissional conseguirá desempenhar a prática assistencialista de parto mais adequada, nos momentos críticos do nascimento e em todos os partos (OMS, 2017; SPECTOR JM, et al., 2013; COUSENS S, et al., 2011).

Com relação aos direitos relacionados a saúde da gestante, o Ministério da Saúde preconiza que a mesma durante o pré-natal deverá no mínimo realizar seis consultas médicas, caso queira pode ter a presença de um acompanhante nas consultas, além do direito ao cartão da gestante onde será anotado 
todas as informações de consultas e exames pré-natais, e será a partir dele que o médico plantonista saberá do quadro desenvolvido durante a gestação (PORTELLA AP, et al., 2000). Além de ter direito a uma atenção humanizada, de qualidade e com preceitos éticos, sendo as condutas aplicadas benéficas para a gestante e o recém-nascido sem a aplicação de intervenções desnecessárias, garantia a privacidade e autonomia da mulher e a sua participação nas decisões das condutas a serem adotadas (VELHO MB, et al., 2010).

Diante disso, esse trabalho buscou verificar, se as gestantes de uma região do estado de São Paulo estão realizando todas as etapas necessárias para o melhor acolhimento no parto, para assim fazer um levantamento de quais pontos precisam ser melhorados ou aprimorados para a implementação de um parto humanizado levando a uma melhor condição para a parturiente e o recém-nascido, além de diminuir assim o custo dos mesmos no hospital, e para os Sistema Único de Saúde (SUS).

\section{MÉTODOS}

Trata-se de uma pesquisa prospectiva exploratória e de natureza descritiva e quantitativa. Para o levantamento de dados sobre o parto seguro, tanto com relação as orientações durante o período de gestação, quanto durante o processo de internação da gestante e trabalho de parto a população do estudo foi constituída de gestantes atendidos e/ou hospitalizados no num hospital do interior de São Paulo, sendo um total de 108 gestantes. Foram excluídas as gestantes que se recusaram a participar da pesquisa e aquelas que tinham idade inferior a 18 anos.

A pesquisa foi desenvolvida em um Hospital Universitário em Bragança Paulista, interior de São Paulo. Este hospital atente pacientes do SUS e particular, sendo referência no atendimento de gestantes de alto risco.

A coleta de dados se submeteu por meio de questionário autoaplicável que continha variáveis como idade, estado civil, escolaridade, período da gestação, frequência de consultas no pré-natal, conhecimento sobre o assunto "Parto Seguro", entre outros. Este questionário foi elaborado pelos autores do artigo baseado em informações contidas na literatura. Após a coleta dos dados, estes foram tabulados, utilizando - Microsoft Excel, e a analisados de forma estatística, utilizando o programa Origin. Os resultados encontrados foram apresentados na forma de porcentagem para ser comparado com dados encontrados na literatura.

Este trabalho foi desenvolvido após aprovação do Comitê de Ética da Instituição, conforme às exigências da Resolução 196/96 do Conselho Nacional de Saúde (Parecer número: 2.908.849). Antes da obtenção desses dados, foi solicitado o preenchimento do Termo de Consentimento Livre e Esclarecido, para toda a população estudada.

\section{RESULTADOS}

O presente trabalho foi desenvolvido a fim de verificar o perfil das gestantes com relação ao parto seguro, onde foram entrevistadas um total de 108 gestantes, que estão distribuídas em idades de 19 a 45 anos. De acordo com a Tabela 1 é possível analisar o perfil sociodemográficos das participantes. Com relação a idade verificou-se que $33,3 \%$ das gestantes têm entre $18-24$ anos, 35,2\% delas estão entre $25-34$ anos e $31,5 \%$ acima de 35 anos. Além disto, mais de $50 \%$ delas são casadas, tem pelo menos o ensino médio completo e trabalham. 
Tabela 1: Perfil sociodemográfico das gestantes entrevistadas em um hospital universitário de Bragança Paulista, interior de São Paulo $n=108$.

\begin{tabular}{ccc}
\hline Categoria & Subcategoria & $\mathbf{n}(\%)$ \\
\hline \multirow{2}{*}{ Idade (anos) } & $18-24$ & $36(33,3)$ \\
& $25-35$ & $38(35,2)$ \\
& 35 ou mais & $34(31,5)$ \\
\hline \multirow{2}{*}{ Estado civil } & Solteira & $21(19,4)$ \\
& Casada & $56(51,9)$ \\
& Divorciada & $3(2,8)$ \\
& Viúva & $0(0)$ \\
& Outro & $28(25,9)$ \\
\hline \multirow{2}{*}{ Escolaridade } & Analfabeta & $1(0,9)$ \\
& Ensino Fundamental Incompleto & $11(10,2)$ \\
& Ensino Fundamental Completo & $8(7,4)$ \\
& Ensino Médio Incompleto & $17(15,7)$ \\
& Ensino Médio Completo & $43(39,8)$ \\
& Ensino Superior Incompleto & $15(13,9)$ \\
& Ensino Superior Completo & $13(12)$ \\
\hline \multirow{2}{*}{ Trabalha } & Sim & $61(56,5)$ \\
& Não & $47(43,5)$ \\
\hline
\end{tabular}

Fonte: Silva EN, et al., 2020.

O hospital onde a pesquisa foi realizada é referência para gestações e partos de alto risco, portanto foi natural encontrar dentre a amostra a maioria gestantes de alto risco, com inúmeras patologias, como hipertensão, diabetes gestacional, trabalho de parto prematuro, entre outros. Porém, durante o estudo, houve casos de gestantes que não pertenciam ao grupo de alto risco, mas estavam em consulta no pronto socorro do Hospital Universitário por conta de um evento adverso, como sangramentos, e dores. Dentre as gestantes consultadas, apenas $1 \%$ não sabiam de sua condição e o motivo que as classificam como gestantes de alto risco. $\mathrm{Na}$ amostra total, $7,41 \%$ estão no $1^{\circ}$ trimestre de gestação, $11,11 \%$ no $2^{\circ}$ trimestre e $81,48 \%$ no $3^{\circ}$ trimestre (Tabela 2).

Tabela 2 - Análise do período de gestação entre as parturientes entrevistadas $n=108$.

\begin{tabular}{cc}
\hline Período (Trimestre) & Resultado (\%) \\
\hline $1^{\circ}$ & 7,41 \\
$2^{0}$ & 11,11 \\
$3^{0}$ & 81,48 \\
\hline
\end{tabular}

Fonte: Silva EN, et al., 2020.

Outro fator importante estudado foi a consulta pré-natal, uma vez que o acompanhamento garante uma gestação saudável e um parto seguro, diminuindo o risco de complicações e mantendo o bem-estar da mãe e do feto. As parturientes analisadas $75,92 \%$ estão entre a 4 e 6 consultas de pré-natal e as demais já realizaram mais de 6 consultas, de acordo com seu período gestacional (Figura 1A), respeitando as recomendações, sendo que apenas $1,85 \%$ das parturientes que estão no 3 ㅇ trimestre, estão com os números de consultas abaixo do que é recomendado pelo Ministério da Saúde. Contudo, como quase todas são gestantes eram de alto risco havia-se a necessidade de saber quantas consultas foram realizadas no Hospital Universitário, sendo $63 \%$ de 1 a 3 consultas pré-natal, $16 \%$ de 4 a 6 consultas e $21 \%$ com mais de 6 consultas (Figura 1B). 
Figura 1 - Distribuição das gestantes analisadas com relação à consulta pré-natal.
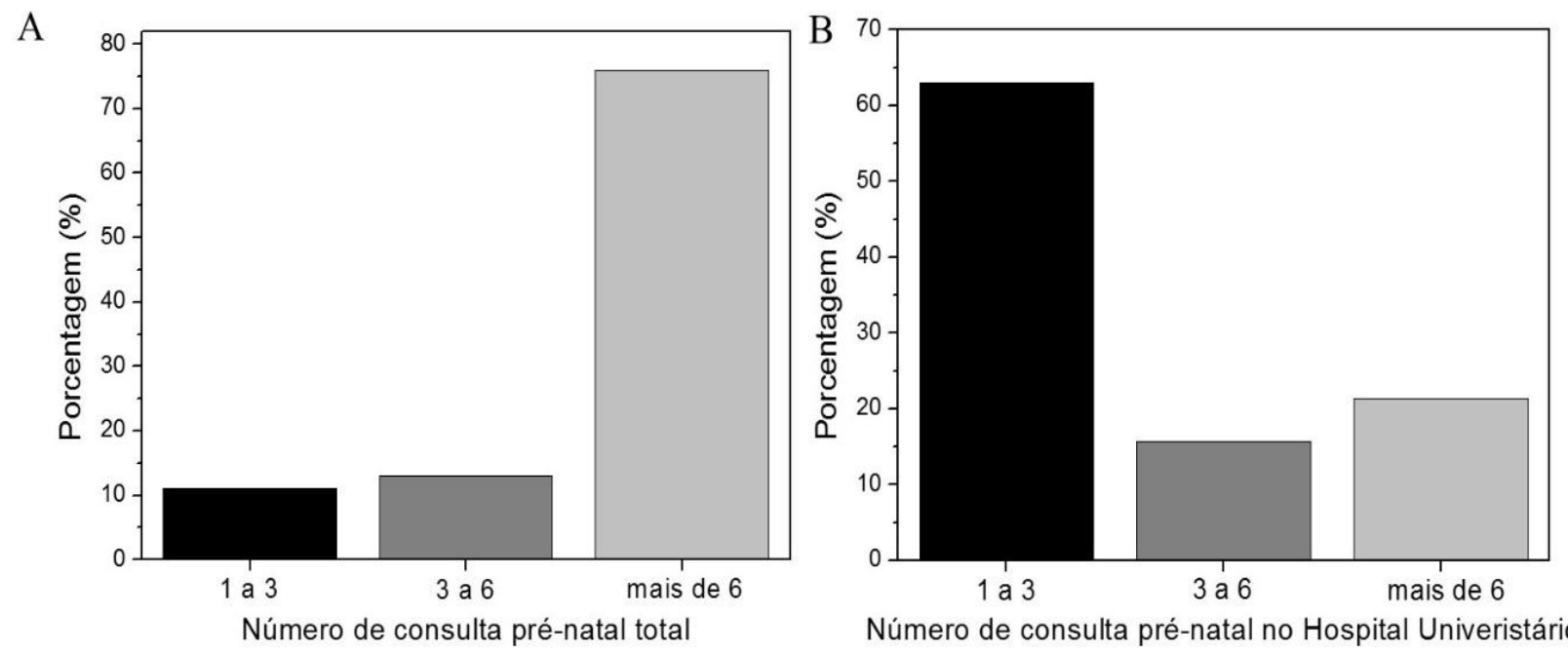

Legenda: (A) gestantes analisadas foram questionadas com relação a quantidade de consulta pré-natal realizada até o momento da entrevista; (B) número realizado no Hospital Universitário .

Fonte: Silva EN, et al., 2020.

No que se diz respeito ao parto seguro, ao analisar os dados obtidos, observa-se que a maioria das gestantes, ou seja, 74,1\%, não sabem o que se define um parto seguro. Apenas $25,9 \%$ gestantes dizem conhecer um pouco sobre o tema, por interesse próprio e a grande maioria o define como sendo "Aquele parto, onde são feitas ações para que seja livre de complicações para a mãe ou para o bebê". Das gestantes que relataram conhecer do assunto $53,6 \%$ tiveram informação com profissional da saúde, sendo que algumas relataram ser por mais de um profissional, as demais gestantes deste grupo $(46,4 \%)$ buscaram informação por conta própria. Estas gestantes que buscaram informação por conta própria $57,1 \%$ têm nível superior e as outras $42,9 \%$ tem nível médio indicando que quanto maior o grau de escolaridade mais informação as gestantes buscam. Em relação as orientações, dentre toda a amostra apenas 2,8\% diz ter recebido as orientações sobre parto seguro por meio do profissional enfermeiro, 13,9\% diz ter recebido orientações pelo médico, 1,8\% por outro profissional, e $81,5 \%$ simplesmente não recebem informações. Vale ressaltar que $13,3 \%$ das gestantes que foram orientadas sobre o parto seguro, não sabiam o que era, portanto é de suma importância no momento da orientação os profissionais utilizem linguajar que seja de fácil compreensão para as gestantes. Apesar desta falta de orientação, 92,6\% da amostra afirmam que durante as consultas com seus médicos e enfermeiros, eles escutam suas queixas e tiram suas dúvidas (Tabela 3).

Tabela 3 - Perfil das informações dadas pelas gestantes com relação ao conhecimento sobre parto seguro e as orientações recebidas pelos profissionais durante a consultas pré-natal.

\begin{tabular}{ccc}
\hline Informação dadas durante a consulta pré-natal & Resposta esperada & $\mathbf{n ~ ( \% )}$ \\
\hline Definição de parto seguro & $\operatorname{Sim}$ & $28(25,9)$ \\
& Não & $80(74,1)$ \\
\hline \multirow{2}{*}{ Recebeu orientação parto seguro } & Sim & $15(13,9)$ \\
& Não & $93(86,1)$ \\
\hline \multirow{2}{*}{ Por qual profissional } & Enfermeiro & $3(2,8)$ \\
& Médico obstetra & $15(13,9)$ \\
& Outro & $2(1,9)$ \\
\hline Os profissionais escutam suas queixas e tiram & Sim & $100(92,6)$ \\
suas dúvidas & Não & $8(7,4)$ \\
\hline
\end{tabular}

Fonte: Silva EN, et al., 2020. 


\section{DISCUSSÃO}

O atendimento de má qualidade durante os nascimentos nos países de baixa e média renda é um dos principais fatores que contribuem para o problema materno e do recém-nascido, mas o progresso tem sido lento na identificação de métodos eficazes para enfrentar essas deficiências em escala. As mulheres são a maioria da população residente no Brasil $(51,9 \%)$ e são as principais usuárias dos serviços de saúde, segundo os dados da Programa Nacional em Saúde - PNS (BRASIL, 2015). De acordo com dados do Instituto Brasileiro de Pesquisa e Geografia a taxa de fecundidade no Brasil era de 2,39 no ano de 2000 e este valor em 2015 era de 1,72, e o de mortalidade infantil no mesmo período foi de 29,02 para 13,82 por mil nascidos vivos, o que demonstra uma diminuição em 15 anos de aproximadamente $28 \%$ da fecundidade e 52,4\% na mortalidade infantil no Brasil, indicando que os programas incrementados nos serviços de saúde estão no caminho certo (BRASIL, 2013).

Dos dados sociodemográficos encontrados $31,5 \%$ das gestantes participantes têm idade acima de 35 anos, $51,9 \%$ são casadas, $56,5 \%$ trabalham e $65,7 \%$ tem pelo menos ensino médio completo demonstrando que as mulheres estão se preparando socialmente e economicamente para este momento. A gravidez e o parto acima dos 35 anos estão em acessão no Brasil e muitas vezes porque estas mulheres querem consolidar suas relações em novas uniões conjugais, à estabilidade financeira e à maturidade do casal. Estes momentos em mulheres desta idade sempre foram cercados de inúmeras dúvidas e mitos, sendo classificada muitas vezes como uma gestação de risco, porém com os avanços tecnológicos e da medicina atual, as gestações estão se tornando cada vez mais seguras em qualquer época da vida reprodutiva da mulher, desde que sejam seguidas as recomendações para um parto seguro, frente a isso faz-se necessário um acompanhamento sistematizado, e uma clínica especializada para descartar qualquer possível intercorrência com a mãe ou bebê (SILVA JLCP, SURITA FGC, 2009; CAETANO LC, et al., 2011; PARADA CMGL, TONETE VLP, 2009; TEIXEIRA EC, et al., 2015).

No Brasil a idade média na qual as mulheres tiveram sua primeira gravidez foi de 21 anos, ao passo que quanto maior sua escolaridade mais tarde isso ocorre, sendo que a mesma situação se observa com relação a fecundidade, sendo que quanto menor a escolaridade maior a fecundidade, as mulheres com menos de 4 anos de estudo tinham 3,7 filhos e com 8 anos ou mais 1,7 filhos (BRASIL, 2015).

O acompanhamento, através da consulta pré-natal, garante uma gestação saudável e um parto seguro, diminuindo o risco de complicações e mantendo o bem-estar da mãe e do feto. O Ministério da Saúde preconiza que sejam realizadas pelo menos 6 consultas, sendo a primeira no primeiro trimestre, duas no segundo e as demais no terceiro trimestre de gestação (BRASIL, 2015). Das gestantes que participaram da pesquisa $81,5 \%$ delas estavam no terceiro trimestre de gestação e todas estavam em dia com o número de consulta pré-natal.

Apenas 25,9\% gestantes dizem conhecer um pouco sobre parto seguro e humanizado, dentre estas $57,1 \%$ tem ensino superior e $42,9 \%$ ensino médio, demonstrando que as mulheres estão se preocupando em saber mais sobre o parto. Além disto, 53,6\% tiveram informação com profissional da saúde, sendo que algumas relataram ser por mais de um profissional e $46,4 \%$ buscaram informação por conta própria, mostrando que as gestantes estão cada vez mais procurando informações que as permitam tomar decisões das condutas a serem tomadas sobre o parto. De acordo com Hotimsky SN, et al. (2002) a realização de parto cirúrgico no Brasil é dito como uma alegação de desejo da parturiente, porém no seu estudo foi comprovado que a cesariana não é uma preferência entre as parturientes, sendo que as entrevistas tinham diferentes faixas etárias e experiências em relação à vida reprodutiva.

Com relação a conduta dos profissionais $92,6 \%$ das gestantes que participaram da pesquisa relatam que os profissionais escutam suas queixas e dúvidas, portanto elas são assistidas neste momento, o que opõem achado de autores que abordam que mulheres de diferentes regiões do país relatam desrespeito e maus tratos, além da indisponibilidade dos profissionais da saúde (HOTIMISKY SN, et al., 2002; da SILVA ALA, et al., 2014). Portanto a humanização da assistência, nas suas muitas versões, expressa uma mudança na compreensão do parto como experiência humana e, para quem o assiste, uma mudança no "que fazer" diante do sofrimento do outro humano (DINIZ CSG, 2005). 
Além disto, dados demonstram que as parturientes buscam se apropriar do saber dos profissionais da saúde para formular suas expectativas e preferências em relação ao parto, portanto há sim um impacto positivo quando existe um suporte emocional, principalmente na diminuição da incidência de parto cesáreo e na duração do trabalho de parto. Esses cuidados de ouvir a parturiente e esclarecer as suas dúvidas associadas com todo os demais cuidados prestados a gestante estão sendo abordado como uma estratégia benéfica para o trabalho de parto e nascimento normal (HOTIMISKY SN, et al, 2002).

Com o advento de recursos tecnológicos, que auxiliou muito no desenvolvimento do bem-estar da parturiente e do recém-nascido, o compromisso do profissional e o relacionamento com a mulher na assistência ao parto foram perdendo-se ao longo dos anos (da SILVA ALA, et al., 2014). Das gestantes que participaram 13,9\% receberam informação sobre o parto seguro de médicos obstetra, $2,8 \%$ de enfermeiros e 1,9\% de outros profissionais da saúde mostrando que a equipe multidisciplinar não está informando as gestantes com relação ao parto seguro. A gestante se sente mais segura quando a enfermeira obstétrica está presente acompanhando todo o desenvolvimento do trabalho de parto. Devido a esta presença constante é possível diagnosticar precocemente intercorrências, o que diminui a chance de problemas com a mãe e o recém-nascido. Através desta conscientização das gestantes as enfeiras obstétricas estão sendo cada vez mais valorizadas (VELHO MB, et al., 2010). Sendo assim é de suma importância que a enfermagem seja mais participativa com relação a esta participação em orientar e informar as gestantes, uma vez que são profissionais que estão em contato direto com elas.

As ações educativas são descritas como eventos presentes no cotidiano da enfermeira, sendo que na área obstétrica, associam-se ao incentivo e apoio às mulheres no ciclo gravídico-puerperal. As enfermeiras orientam sobre o pré-parto, evolução do trabalho de parto, contrações uterinas, o posicionamento de escolha da gestante, incentivo ao nascimento de parto normal e suas facilidades no cuidado ao recémnascido. Como ações educativas das enfermeiras foram identificadas a apresentação do local de permanência da mulher no pré-parto, parto e puerpério; orientações a respeito da liberdade de escolha nas diferentes modalidades de atenção ao parto, tanto em relação ao local quanto à posição; escolha do método de cuidado e conforto nos momentos que antecedem o nascimento e disponibilidade para o esclarecimento de dúvidas. Esses aspectos fazem com que a mulher se tranquilize e se sinta valorizada, o que facilita a evolução do parto (FERNANDES BM, et al., 2011).

Frente a isso, surge a necessidade de analisar melhor as informações dadas as essas gestantes durante as consultas, por exemplo sobre seus direitos e deveres, e principalmente levantar a importância de abordar assuntos ainda não colocados em pauta, mas que já se fazem fundamentais na vivencia do pré-natal, como no caso o parto seguro. Além disto, auxiliar no processo de conscientização da equipe de enfermagem do quanto ela é importante em ações educativas, que levarão a uma menor mortalidade da parturiente e do recém-nascido.

\section{CONCLUSÃO}

A partir dos resultados encontrados é possível concluir que existe a necessidade de reformular as informações dadas as gestantes durante as consultas pré-natal, como por exemplo, reforçar sobre seus direitos e deveres, e principalmente abordar assuntos ainda não colocados em pauta, mas que já se fazem fundamentais na vivencia do pré-natal, como no caso o parto seguro. Ainda, faz-se necessário encontrar estratégias para o desenvolvimento de atitudes tanto das gestantes quanto dos profissionais da saúde com relação a implementação de um parto seguro, assim levando a uma diminuição no número de óbitos de gestantes e recém-nascidos, além da diminuição do tempo de estada deles no hospital.

\section{REFERÊNCIAS}

1. BRASIL - Ministério do Planejamento, Orçamento e Gestão; Instituto Brasileiro de Geografia e Estatística; Diretoria de Pesquisa; de Trabalho e Rendimento. Pesquisa nacional de saúde 2013: Ciclos de vida - Brasil e grandes regiões / IBGE, Coordenação de Trabalho e Rendimento. Rio de Janeiro: IBGE, 2015, 90 p. 
2. BRASIL - Instituto Brasileiro de Geografia e Estatística (IBGE). Projeção da população do Brasil - 2013. 2013. Disponível em: https://brasilemsintese.ibge.gov.br/populacao. Acesso em: 08 de jun 2019.

3. CAETANO LC, et al. Gravidez depois dos 35 anos: uma revisão sistemática da literatura. Revista Mineira de Enfermagem, 2011;15(4):579-87.

4. COUSENS S, et al. National, regional, and worldwide estimates of stillbirth rates in 2009 with trends since 1995: a systematic analysis. Lancet, 2011; 377:1319-30.

5. da SILVA ALA, et al. Avaliação da assistência hospitalar materna e neonatal: índice de completude da qualidade. Revista Saúde Pública, 2014; 48(4):682-691.

6. DINIZ CSG. Humanização da assistência ao parto no Brasil: os mitos sentidos de um movimento. Ciência e Saúde Coletiva, 2005; 10(3):627-37.

7. FERNANDES BM, et al. Avaliação do pré-natal habitual realizado por enfermeiras obstetras de uma casa de parto. Revista de Enfermagem do Centro Oeste Mineiro, 2011; 1(1):17-29.

8. GAWANDE A. The checklist manisfeto: How to get things right. London Journal of Primary Care, 2010; 3(2):124.

9. HOTIMISKY SN, et al. O parto como eu vejo... ou como eu o desejo? Expectativas de gestantes, usuárias do SUS, acerca do parto e da assistência obstétrica. Cadernos de Saúde Pública, 2002; 18(5):1303-11.

10. ORGANIZAÇÃO MUNDIAL DA SAÚDE (OMS). Guia de implementação da lista de verificação da OMS para partos seguros: melhorar a qualidade dos partos realizados em unidades de saúde para as mães e recém-nascidos [WHO safe childbirth checklist implementation guide: improving the quality of facility-based delivery for mothers and newborns]. Genebra: Ariadne Labs; 2017, 67p.

11. PARADA CMGL, TONETE VLP. Experiência da gravidez após os 35 anos de mulheres com baixa renda. Escola Anna Nery, 2009; 13(2):385-92.

12. PORTELLA AP, et al. Gravidez saudável e parto seguro são direitos da mulher. São Paulo: Rede Nacional Feminista de Saúde e Direitos Reprodutivos, Ministério da Saúde; 2000, 11p.

13. RISCADO LC, et al. A decisão pela via de parto no Brasil: temas e tendências na produção da saúde coletiva. Texto e Contexto Enfermagem, 2016; 25(1):1-10.

14. SILVA JLCP, SURITA FGC. Idade Materna: resultados perinatais e via de parto. Revista Brasileira de Ginecologia e Obstetrícia, 2009; 31(7):321-5.

15. SPECTOR JM, et al. Designing the WHO safe childbirth checklist program to improve quality of care at childbrith. International Journal of Gynecology \& Obstetrics, 2013; 122(2):164-8.

16. TEIXEIRA EC, et al. Gravidez em mulheres acima de 34 anos no Brasil - análise da frequência entre 2006 e 2012. Revista HUPE, 2015; 14(1):6-11.

17. VELHO MB, et al. Reflexões sobre a assistência de enfermagem prestada à parturiente. Revista Brasileira de Enfermagem, 2010; 63(4):652-9. 